\title{
Adjuvant Effect of Leuprolide on Stroke: A Case report
}

\section{Carlo Sebastiano Tadeo ${ }^{a}$, Barbara Incorvaia ${ }^{a}$, Raffaela Russo $^{a}$, Luciana De Lauretis $^{\mathrm{b}}$}

\footnotetext{
a Stroke Unit, Clinical Institute Città Studi, Milan, Italy

${ }^{\mathrm{b}}$ Gynaecology Department, Clinical Institute Città Studi, Milan, Italy
}

\begin{abstract}
Objectives: To contribute to current knowledge on the vascular risk of oestrogens.

Materials and methods: A 44-year-old woman received a $11.25 \mathrm{mg}$ Leuprolide exteneded release injection to control bleeding from a $7 \mathrm{~cm}$ uterine fibroid tumour; 45 days later, she had a stroke due to right frontal lobe ischaemia. Thrombolysis induced complete remission. Three years previously, while taking a birth control pill, the patient had suffered from a stroke that involved her left temporal lobe. She was heterozygous for Factor V R2 H1299R locus and homozygous for the 4G/4G mutation of the PAI-1 gene. Even though her homocysteine level was normal, the patient was homozygous for the MTHFR C677T mutation and although she had never had severe bleeding, she was also homozygous for Factor XIII V34L.
\end{abstract}

Results and conclusion: This patient's prothrombotic condition could have been enhanced by leuprolide since its stimulatory effect on oestrogen production would still have been minimally present at the time of cerebral thrombosis.

Keywords: Leuprolide, stroke, thrombosis, thrombolysis, coagulation abnormalities, LHRH analogues

Received: $19 / 01 / 2014$

Accepted: $15 / 10 / 2014$

Published: $11 / 11 / 2014$

How to cite this article: Tadeo SC, Incorvaia B, Russo R, De Lauretis L. Adjuvant Effect of Leuprolide on Stroke: A Case report. EJCRIM 2014;1:doi: 10.12890/2014_000061

Conflicts of Interests: The authors declare that they have no conflicts of interest related to this research.

\section{Introduction}

Stroke in young subjects is devastating. A structured programme of interventions has recently been implemented to prevent it, to dissolve the thrombus during the acute phase before the ischaemia becomes irreversible and to avoid the thrombogenic adverse effects of drugs and procedures [1]. The present case addresses the latter type of effective interventions. We herein describe a patient who suffered a stroke due to the administration of an LHRH analogue given to curb chronic minor bleeding from a fibroid tumour of the uterus. 


\section{Case description}

A 44-year-old woman had a sudden onset of dysarthria, left faciobrachial motor weakness and limb ataxia (NIHSS=5). On admission to hospital, an emergency brain CT scan showed two old foci of ischaemia in her left parietal area and right cerebellum. Thrombolysis was performed according to the established guidelines with rtPA $0.9 \mathrm{mg} / \mathrm{kg}$ [1]. The symptoms abated progressively during the ensuing four days. The patient was in sinus rhythm. A cerebral angiography performed the day after thrombolysis disclosed no vascular obstruction, while an NMR 2 days later showed an ischaemic change involving her right cortical and subcortical frontal sylvian area. Trans-oesophageal echocardiogram and colour-flow duplex ultrasound examinations ruled out cardiac and aortic arch abnormalities as well as carotid plaques. Past history revealed that the patient had been chronically bleeding from a large $7 \mathrm{~cm}$ uterus fibroid tumour that had been treated 45 days previously with an $11.25 \mathrm{mg}$ Leuprolide Depot i.m. injection. Her former stroke in her left parietal area had occurred 3 years earlier while she was taking an oestro-progestinic birth control medication. During the present event, her homocysteine blood level was $5.6 \mu \mathrm{M} / \mathrm{l}$, D-dimer $511 \mathrm{ng} / \mathrm{ml}$ (it fell to 144 in 4 days), C3c $79 \mathrm{mg} / \mathrm{dl}$, progesterone $<0.2 \mathrm{mU} / \mathrm{ml}, 17 \beta$-oestradiol $107.5 \mathrm{pg} / \mathrm{ml}$, LH (Luteinizing hormone) $3.7 \mathrm{mUl} / \mathrm{ml}$, FSH (Follicle stimulating hormone) $6.6 \mathrm{mUl} / \mathrm{ml}, \beta-H C G$ (Human chorionic gonadotropin) $<0.1 \mathrm{mUl} / \mathrm{ml}, \mathrm{Hb}$ (Hemoblobin) $8.5 \mathrm{~g} / \mathrm{dl}$, ANA (Antinuclear antibodies) test and LAC (Lupus anticoagulant) negative, coagulation protein C 59\%, free protein S $172 \%$, serum iron $42 \mu \mathrm{g} / \mathrm{dl}$, transferrin $247 \mathrm{mg} / \mathrm{dl}$ and ferritin $16 \mathrm{ng} / \mathrm{ml}$. Her home medications were aspirin $100 \mathrm{mg}$ q.d., folic acid $5 \mathrm{mg}$ q.d. and iron sulphate $300 \mathrm{mg}$ p.o., b.i.d. She was transfused with packed red cells to a $\mathrm{Hb}$ of $10.5 \mathrm{~g} / \mathrm{dl}$.

An exhaustive screening for prothrombotic abnormalities revealed the presence of the heterozygous mutation H1299R of coagulation Factor $\mathrm{V}$, the homozygous mutation $4 \mathrm{G} / 4 \mathrm{G}$ of PAI-1 and the homozygous mutation C677T of MTHR. The patient was also a carrier of the homozygous mutation C34L of coagulation Factor XIII.

\section{Discussion}

This patient was at risk of stroke as she had already experienced three ischaemic episodes before age 45 . The first two ischaemic events were presumably due to the homozygous deficit of MTHFR, which preferentially affects arterial, mainly cerebral, vessels, although the alteration of Factor V. The oestro-progestin combination could have played an adjuvant role. However, this most recent stroke occurred in the presence of a normal homocysteine serum level, presumably due to the daily prophylaxis with folic acid, thus ruling out MTHFR as a causative factor.

After a Food and Drug Administration (FDA) review, LHRH analogues were introduced into the market without warning against the occurrence of stroke. The post-marketing clinical experience of these drugs found a small, approximately $1 \%$, albeit statistically significant incidence of stroke, mainly in patients with diabetes mellitus, cardiovascular disease and previous cerebral ischaemia. ${ }^{1}$ The literature mentions mostly male patients treated with chemical castration for prostate cancer. In contrast, our patient is a female treated for uterine bleeding caused by a fibroid tumour, one of the indications listed for LHRH analogue use [2]. These drugs may cause thrombosis because of the sudden upsurge of oestradiol and progestin levels lasting 10 days after beginning their administration. Although the ischaemic event occurred in our patient long after the initial oestrogen upsurge had faded away (i.e., 45 days after the administration of

\footnotetext{
${ }^{1}$ FDA warning on 20 October 2010, in which manufacturers "transient ischemic attack and paralysis" in the drug data sheet. This was due to the results of an FDA investigation into these products.
} 
leuprolide), oestrogen levels would still have been elevated. It is, therefore, reasonable to hypothesize that the GHRH still had a prothrombotic effect.

In a recent systematic review, it was proposed that the prolonged supraphysiological levels of oestrogen, released by high-dose pellet implants, were responsible for the detrimental effects of oestrogen. However, it also seems plausible that the LHRH analogues presently marketed could act by some unknown direct effect on cerebral vessels of patients who have already suffered from cerebral ischaemia, or who are affected by vascular and/or cardiovascular diseases, like diabetes. In fact, Fujiki and co-workers claim such a mechanism for the leuprolide-associated stroke in a young Japanese woman [3], suggesting the enhancement of vascular production of oxygen radicals. A similar, direct vascular effect was demonstrated in a case of myocardial infarction [4]. Although the mechanism of action of LHRH analogues in causing brain ischemia must be considered unknown, the risks endowed with their administration must be considered real. It therefore seems prudent to limit the administration to subjects without diabetes, cardiovascular disease, previous ischaemic events and coagulation abnormalities favouring thrombosis.

\section{Learning Points}

- Leuprolide could be a risk factor for ischaemic stroke.

- Genetic prothrombotic abnormalities should be looked for in young patients with cryptogenic strokes.

- It seems prudent to avoid leuprolide use in subjects with diabetes, cardiovascular disease, previous ischaemic events and coagulation abnormalities favouring thrombosis.

\section{References}

1. Van der Worp HB, Van Gujn J. Acute ischemic stroke, N Engl J Med 2007;357:572-579.

2. National Institute for Health and Care Excellence. Heavy menstrual bleeding, NICE guidelines [CG44]. London: RCOG Press, January 2007.

3. Fujiki F, Tsuboi Y, Yamada T. Stroke associated with LH-RH analogue (leuprelin) administration in a young woman, Rinsho Shinkeigaku 2007;47:234-236.

4. Coli S, Magnoni M, Melisurgo G. Myocardial infarction complicating the initial phase of an ovarian stimulation protocol, Int J Cardiol 2007;115:e56-e57. 